\title{
Anticipating Synchronization Based on Optical Injection-Locking in Chaotic Semiconductor Lasers
}

\author{
Kenji Kusumoto and Junji Ohtsubo, Member, IEEE
}

\begin{abstract}
Numerical studies for anticipating chaos synchronization in semiconductor lasers with optical feedback are presented. Anticipating chaos synchronization in a delay-differential system is believed to occur when all chaos parameters between the two systems are perfectly coincident with each other. However, we find new schemes of anticipating chaos synchronization when the parameters between the two systems have mismatches. Under these conditions, the time lag between the two laser outputs is equal to that of anticipating chaos synchronization, but the physical origin of the phenomenon comes from optical injection-locking or amplification in laser systems. We show the evidence of such chaotic synchronization using trajectories in the phase space of the phase difference and the carrier density in the laser oscillations.
\end{abstract}

Index Terms-Chaos, optical feedback, optical injection, semiconductor lasers, synchronization.

\section{INTRODUCTION}

$\mathbf{S}$ YNCHRONIZATION of chaotic oscillations in coupled nonlinear systems is an important issue not only in basic chaos research but also in applications of chaos for secure data transmissions and communications. As chaotic nonlinear devices, the semiconductor laser is suitable for practical applications, since we can make compact and fast-response chaotic systems using semiconductor lasers. A semiconductor laser (edge-emitting narrow stripe type) is a stable laser by its solitary oscillation, however it is easily destabilized by the introduction of extra degrees of freedom, such as injection current modulation, optical injection, and external optical feedback. Among possible external perturbations to induce chaos in semiconductor lasers, external optical feedback has been frequently used to make chaotic lasers and the chaotic dynamics in such a system have been extensively studied. [1]

Chaos synchronization in semiconductor lasers with optical feedback has been studied and it has also been applied to chaotic secure communications. [2] A system of semiconductor lasers with optical feedback is modeled by delay-differential equations. In such systems, there are two types of chaos synchronization; one is complete chaos synchronization in which the two systems, transmitter and receiver systems, are mathematically described by an equivalent set of delay-differential equations and the two systems exhibit entirely almost the same chaotic outputs when a small portion of a transmitter signal is sent to

Manuscript received April 21, 2003; revised August 8, 2003.

The authors are with the Faculty of Engineering, Shizuoka University, Hamamatsu 432-8561, Japan.

Digital Object Identifier 10.1109/JQE.2003.819558 the receiver [3]-[5]. In complete chaos synchronization, the receiver system outputs the signal in advance of receiving the transmitter signal, therefore the scheme is sometimes called anticipating chaos synchronization. Indeed, complete chaos synchronization has already been reported in experimental systems of semiconductor lasers with optical feedback. [4] The other is so-called generalized synchronization of chaos based on phenomena of injection-locking characteristics and signal amplification in nonlinear systems. In this case, the receiver system responds immediately after receiving a signal from the transmitter [6]-[12]. Since the allowance of parameter mismatches for chaos synchronization in the nonlinear systems is not severe compared with the case of complete chaos synchronization, many experimental observations for generalized chaos synchronization in the nonlinear systems have been reported [13]-[18]. The two schemes can be easily distinguished by investigating a time lag between the transmitter and receiver signals. Complete chaos synchronization with anticipating time lag is a typical feature in nonlinear delay-differential systems. Anticipating chaos synchronization has also been observed in systems of incoherent optical feedback [19], [20] and optoelectronic feedback [21], [22]. However, generalized synchronization of chaotic oscillations has never been found in these systems, since injectionlocking is not possible. It is noted that delay-differential conditions are not essential for complete chaos synchronization, and continuous systems (such as the Lorenz system) that are described by differential equations with more than three variables have a solution of complete chaos synchronization [23]. The investigation of the origin of chaos synchronization in various nonlinear systems is important for applications of chaotic secure communications, since the system configurations and mechanisms affect the robustness and the degree of the security in communication systems.

In this paper, we numerically investigate regions of chaos synchronization in the phase space of the frequency detuning between transmitter and receiver lasers and the optical injection rate in the systems of semiconductor lasers with optical feedback. Besides the already reported results, we observe a new type of anticipating chaos synchronization scheme when the optical feedback rate in the transmitter laser is high enough. In these regions, a time lag between the transmitter and receiver lasers obeys anticipating chaos synchronization, while the origin of the synchronization comes from optical injectionlocking. The evidence of the phenomena is shown by using chaotic trajectories in the phase space of the phase difference and the carrier density of the laser oscillation. 


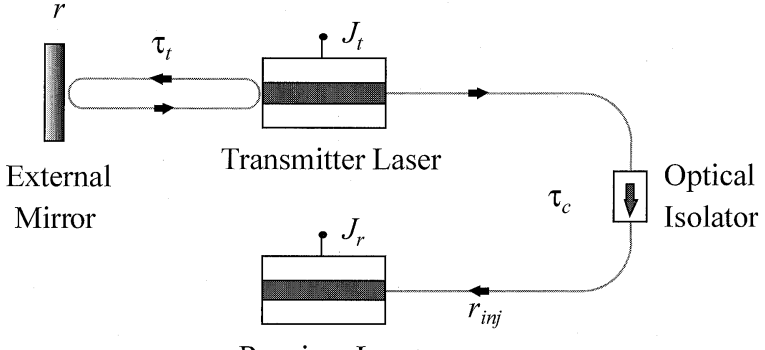

Receiver Laser

Fig. 1. Schematic diagram of a chaos synchronization system in semiconductor lasers with optical feedback. The model is an open-loop system in which only the transmitter laser has external optical feedback.

\section{MODEL OF THE SYSTEM}

We consider chaos synchronization in semiconductor laser systems with optical feedback. The model under consideration is shown in Fig. 1. We prepare two semiconductor lasers having almost the same device characteristics as light sources. The transmitter laser system has an optical feedback loop, whereas the receiver system has no optical feedback loop (we refer to this type of system as an open-loop system in the following). The transmitter laser exhibits chaotic oscillations depending on the feedback parameters. With chaotic signal injection from the transmitter to the receiver, the receiver laser synchronizes with the transmitter laser under appropriate conditions.

The systems are described by the following set of rate equations for the laser fields $E_{t, r}$, the phases $\varphi_{t, r}$, and the carrier densities $n_{t, r}$. [2] The subscripts $t$ and $r$ stand for the transmitter and receiver lasers. For the transmitter we have

$$
\begin{aligned}
\frac{d E_{t}(t)}{d t} & =\frac{1}{2} G_{n, t}\left\{n_{t}(t)-n_{\mathrm{th}, t}\right\} E_{t}(t)+\frac{\kappa_{t}}{\tau_{\mathrm{in}}} E_{t}\left(t-\tau_{t}\right) \cos \theta_{t}(t) \\
\frac{d \varphi_{t}(t)}{d t} & =\frac{1}{2} \alpha_{t} G_{n, t}\left\{n_{t}(t)-n_{\mathrm{th}, t}\right\}-\frac{\kappa_{t}}{\tau_{\mathrm{in}}} \frac{E_{t}\left(t-\tau_{t}\right)}{E_{t}(t)} \sin \theta_{t}(t) \\
\frac{d n_{t}(t)}{d t} & =\frac{J_{t}}{e d}-\frac{n_{t}(t)}{\tau_{s, t}}-G_{n, t}\left\{n_{t}(t)-n_{0, t}\right\}\left|E_{t}(t)\right|^{2} \\
\theta_{t}(t) & =\omega_{0, t} \tau_{t}+\phi_{t}(t)-\phi_{t}\left(t-\tau_{t}\right)
\end{aligned}
$$

and for the receiver we have

$$
\begin{aligned}
\frac{d E_{r}(t)}{d t}= & \frac{1}{2} G_{n, r}\left\{n_{r}(t)-n_{\mathrm{th}, r}\right\} E_{r}(t) \\
& +\frac{\kappa_{\mathrm{inj}}}{\tau_{\text {in }}} E_{t}\left(t-\tau_{c}\right) \cos \xi(t) \\
\frac{d \varphi_{r}(t)}{d t}= & \frac{1}{2} \alpha_{r} G_{n, r}\left\{n_{r}(t)-n_{\mathrm{th}, r}\right\} \\
& -\frac{\kappa_{\mathrm{inj}}}{\tau_{\text {in }}} \frac{E_{t}\left(t-\tau_{c}\right)}{E_{r}(t)} \sin \xi(t) \\
\frac{d n_{r}(t)}{d t}= & \frac{J_{r}}{e d}-\frac{n_{r}(t)}{\tau_{s, r}}-G_{n, r}\left\{n_{r}(t)-n_{0, r}\right\}\left|E_{r}(t)\right|^{2} \\
\xi(t)= & \omega_{0, t} \tau_{c}+\phi_{r}(t)-\phi_{t}\left(t-\tau_{c}\right)-\Delta \omega t
\end{aligned}
$$

where $G_{n, j}(j=t, r)$ is the linear gain coefficient, $n_{\mathrm{th}, j}$ is the threshold carrier density, $n_{0, j}$ is the carrier density at transparency, $\alpha_{j}$ is the linewidth enhancement factor, $\kappa_{t}$ is the feedback coefficient, $\kappa_{\mathrm{inj}}$ is the injection coefficient from the transmitter to the receiver, $J_{j}$ is the bias injection current, $e$ is the electron charge, and $d$ is the thickness of the active area. $\tau_{\text {in }}$ is the flight time of light in the internal laser cavity, $\tau_{t}$ is the round-trip time in the external cavity, $\tau_{s, j}$ is the carrier lifetime, and $\tau_{c}$ is the transmission time of light from the transmitter to the receiver. $\omega_{0, j}$ is the laser frequency at solitary oscillation and $\Delta \omega=\omega_{0, t}-\omega_{0, r}$ is the angular frequency detuning between the two lasers. For small optical feedback, we only consider the effect of a single round trip of light in the external cavity, and the feedback coefficient $\kappa_{t}$ is written as

$$
\kappa_{t}=\left(1-r_{0}^{2}\right) \frac{r}{r_{0}}
$$

where we assumed that the amplitude reflectivities $r_{0}$ for the front and back facets of the laser cavity are the same. It is not always true for practical lasers but the other cases can be calculated in a straightforward manner. $r$ is the reflectivity of the external mirror. The second terms on the right-hand side of (1) and (2) are the external feedback effects. The second terms on the right-hand side of (5) and (6) are the effects of the optical injection from the transmitter to the receiver. When a symmetrical system that also has a feedback loop in a receiver system is employed (we refer to this system as closed loop system), the model is easily extended by simply adding the external feedback terms to (5) and (6).

As origins of chaos synchronization, there are two types of the schemes. One is known as complete chaos synchronization. In this scheme, the rate equations for the receiver laser are mathematically described by the equivalent delay-differential equations as those for the transmitter laser [2]. The conditions are

$$
\begin{aligned}
E_{r}(t) & =E_{t}(t-\Delta t) \\
\phi_{r}(t) & =\phi_{t}(t-\Delta t)-\omega_{0} \Delta t(\bmod 2 \pi) \\
n_{r}(t) & =n_{t}(t-\Delta t) \\
\kappa_{r} & =\kappa_{\mathrm{inj}} \\
\Delta \omega & =0 \\
\Delta t & =\tau_{c}-\tau_{t} .
\end{aligned}
$$

Under the above conditions, the rate equations of the transmitter and receiver lasers are mathematically described by the equivalent equations and the receiver laser can synchronize with the transmitter laser by a chaotic signal transmission from the transmitter laser. In this case, the receiver laser anticipates the chaotic output of the transmitter and it outputs the signal before a time $\tau_{t}$ in advance as understood from (15), so that the scheme is also called anticipating synchronization. As already discussed in the previous paper, the complete chaos synchronization was achieved in a region of low optical injection fraction close to zero-frequency detuning [12].

The other type of synchronization is originated from a wellknown optical injection-locking scheme in laser systems. An optically injected laser in the receiver system will synchronize with the transmitter laser based on the effects of optical injection-locking or amplification due to optical injection from a different light source. The optical injection-locking phenomena in semiconductor lasers depend on the detuning between the frequencies of the transmitter and receiver lasers and the injection rate from the transmitter to the receiver. The injection-locking 
region in the phase space of the frequency detuning and the injection rate is usually located in large injection fraction of several tens of percents in the amplitude with wide range of the frequency detuning. The relation between the two laser fields at this synchronization is written by [2]

$$
E_{r}(t) \propto E_{t}(t-\Delta t)
$$

where $\Delta \tau=\tau_{c}$. Namely, the receiver laser responds immediately after it receives a chaotic signal from the transmitter, since the receiver signal always has a time delay with respect to the transmitter signal. This scheme is sometimes called generalized synchronization of chaotic oscillations to distinguish it from complete chaos synchronization. We can easily distinguish generalized synchronization from complete case by observing the time lag between chaotic signals from the transmitter and receiver lasers.

As we will discuss in the following, all of the conditions from (10)-(14) are not always necessary for anticipating chaos synchronization. Indeed, there is a case of anticipating chaos synchronization only satisfying the condition of (10). In that case, the synchronization comes from optical injection-locking phenomenon as a physical origin, but the transmitter and receiver lasers show output signals corresponding to anticipating chaos synchronization.

\section{ChAOS SYNCHRONIZATION}

Regions of chaos synchronization are numerically investigated in the phase space of the frequency detuning between the transmitter and receiver lasers and the injection fraction from the transmitter to the receiver lasers. In the numerical simulations, the two lasers are assumed to have the same characteristics, and we used the parameter values as $G_{n, j}=8.40 \times 10^{-13} \mathrm{~m}^{3} \mathrm{~s}^{-1}$, $n_{\mathrm{th}, j}=2.18 \times 10^{24} \mathrm{~m}^{-3}, n_{0, j}=1.40 \times 10^{24} \mathrm{~m}^{-3}, \alpha_{j}=3.0$, $e=1.60 \times 10^{-9} \mathrm{C}, V=d w l=1.20 \times 10^{-16} \mathrm{~m}^{3}(V$, $w$, and $l$ are the total volume, width, and length in the active region), $\tau_{s, j}=2.04 \mathrm{~ns}, \tau_{\mathrm{in}, j}=8.0 \mathrm{ps}, r_{0}=0.555$, and $f_{0, t}=\omega_{0, t} / 2 \pi=384.615 \mathrm{THz}$. The bias injection currents were $J_{t}=J_{r}=1.3 J_{\text {th }}$ (where $J_{\text {th }}$ is the threshold injection current). The round-trip time of light within the external cavity was $\tau_{t}=1.0 \mathrm{~ns}$. Without the loss of generality, the time of light transmission from the transmitter to the receiver laser was set to be $\tau_{c}=0$. The reflectivity of the external mirror $r$, the frequency detuning $\Delta f=\Delta \omega / 2 \pi$, and the optical injection rate $r_{\text {inj }}$ from the transmitter to the receiver laser were the variable parameters to investigate chaos synchronization. In the numerical calculations, the optical frequency $f_{0, t}$ of the transmitter laser was fixed and the frequency $f_{0, r}=\omega_{0, r} / 2 \pi$ of the receiver laser was changed when we considered the detuning.

To evaluate the quality of synchronization between output signals from the two lasers for the variations of the variable parameters, we used the following correlation coefficient:

$$
\begin{aligned}
& C(\Delta t)= \\
& \frac{\left\langle\left\{E_{t}(t-\Delta t)-\left\langle E_{t}(t-\Delta t)\right\rangle\right\}\left\{E_{r}(t)-\left\langle E_{r}(t)\right\rangle\right\}\right\rangle}{\sqrt{\left\langle\left\{E_{t}(t-\Delta t)-\left\langle E_{t}(t-\Delta t)\right\rangle\right\}^{2}\right\rangle\left\langle\left\{E_{r}(t)-\left\langle E_{r}(t)\right\rangle\right\}^{2}\right\rangle}} .
\end{aligned}
$$

$\Delta \tau=\tau_{c}-\tau_{t}$ for the case of evaluating anticipating chaos synchronization, while $\Delta \tau=\tau_{c}$ for generalized chaos synchro-

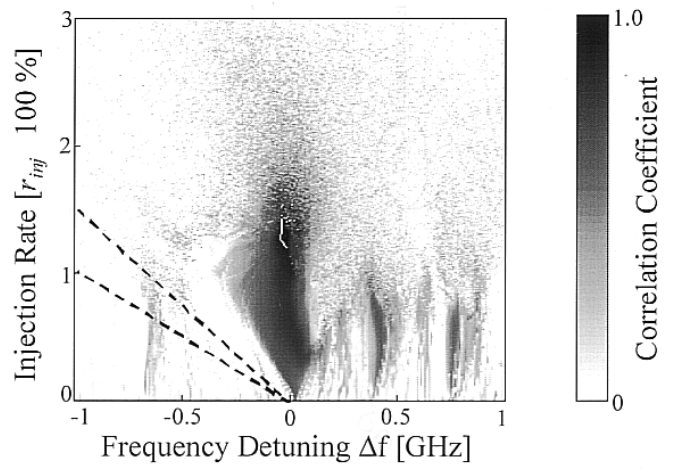

(a)

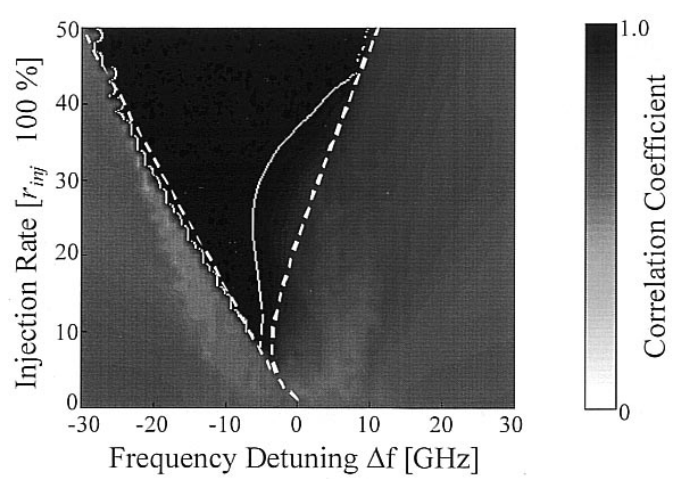

(b)

Fig. 2. Regions of chaos synchronization in the phase space of the frequency detuning $\Delta f$ and the optical injection rate $r_{\text {inj }}$ for weak optical feedback of $r=0.014$. (a) Anticipating chaos synchronization. (b) Generalized chaos synchronization induced by the injection-locking regime. Broken lines show the boundary of the stable injection-locking region. The regions within the thin solid lines represent those for excellent chaos synchronization with a correlation coefficient higher than 0.94 .

nization. For either case, we regarded that successful synchronization was attained when the correlation coefficient exceeded the value of 0.940

Two types of chaos synchronization have already been reported for the variations of the frequency detuning and the optical injection rate. Fig. 2 plots regions of chaos synchronization in the phase space of the frequency detuning and the optical injection rate when the external mirror has a small optical feedback reflectivity of $r=0.014$. Fig. 2(a) shows a case of anticipating chaos synchronization. Anticipating chaos synchronization is realized within the black region around the injection rate of $r_{\mathrm{inj}}=0.014$ at zero-frequency detuning, and this is the case for complete chaos synchronization. The broken line shows the boundary of the region for stable optical injection-locking when the receiver laser is injected by the transmitter laser without external optical feedback. The complete chaos synchronization is achieved in the unstable injection-locking region. On the other hand, generalized chaos synchronization is attained at rather larger injection fraction with a wide range of the frequency detuning as shown in Fig. 2(b). The broken line is also the boundary for stable injection-locking and chaos synchronization is attained within the thin solid line. These results are quite consistent with the previous ones [12].

We also investigated chaos synchronization when the optical feedback rate was very high. The result for an optical feed- 


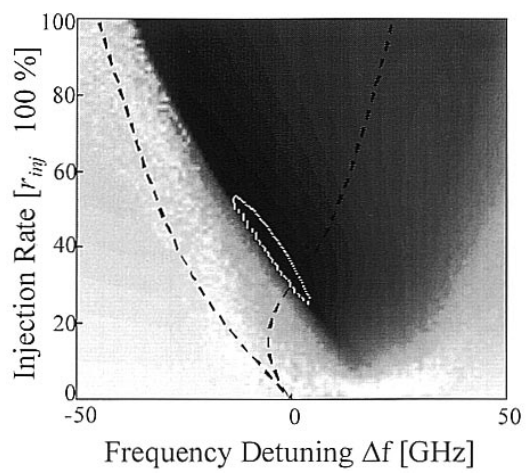

(a)

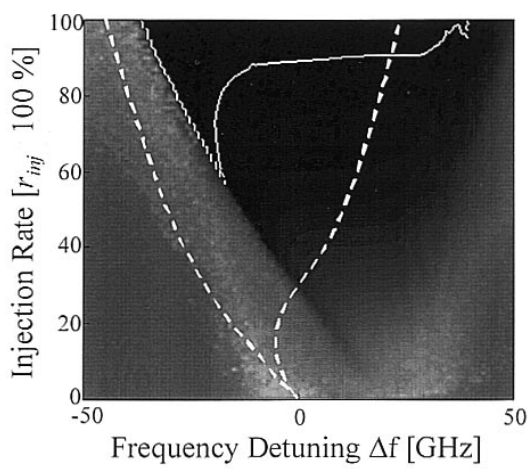

(b)

Fig. 3. Region of chaos synchronization in the phase space of the frequency detuning $\Delta f$ and the optical injection rate $r_{i n j}$ for strong optical feedback of $r=0.300$. (a) Anticipating chaos synchronization. (b) Generalized chaos synchronization induced by an injection-locking regime.

back rate of $r=0.300$ is shown in Fig. 3. Fig. 3(a) shows the region of anticipating chaos synchronization. Fig. 3(b) is a case of generalized chaos synchronization. When an optical feedback in the transmitter laser is strong enough, anticipating chaos synchronization with injection-locking origin is realized only under a strong optical injection condition. Furthermore, anticipating chaos synchronization is attained even for nonzerofrequency detuning, which is a different result from previous one [Fig. 2(a)]. As we will discuss in the next section, anticipating chaos synchronization does not always mean complete chaos synchronization. Namely, the different origin of anticipating synchronization from the complete chaos synchronization scheme exists.

\section{ANTICIPATING SYNCHRONIZATION}

In this section, we focus on anticipating chaos synchronization in the system when the external optical feedback in the transmitter laser is strong. Fig. 4 shows regions of anticipating chaos synchronization for strong optical feedback. The optical injection fractions were $0.200,0.242$, and 0.300 from Fig. 4(a)-(c), respectively. Regions of high correlation coefficient lie along a negative slop in the phase space and expand as the increase of the fraction of optical feedback. For a rather lower external optical feedback [Fig. 4(a)], anticipating chaos synchronization is realized in the unstable injection-locking region. On the other hand, it expands to the stable injection-locking region for the increase of the external

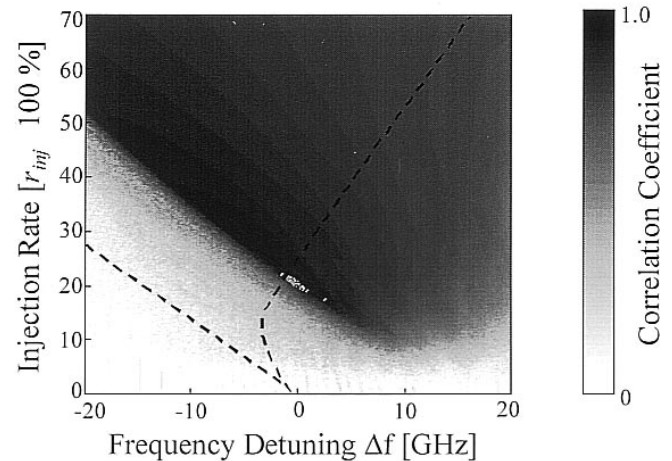

(a)

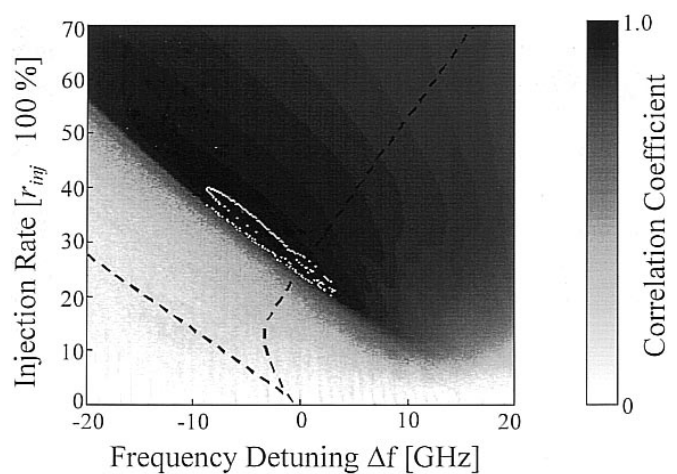

(b)

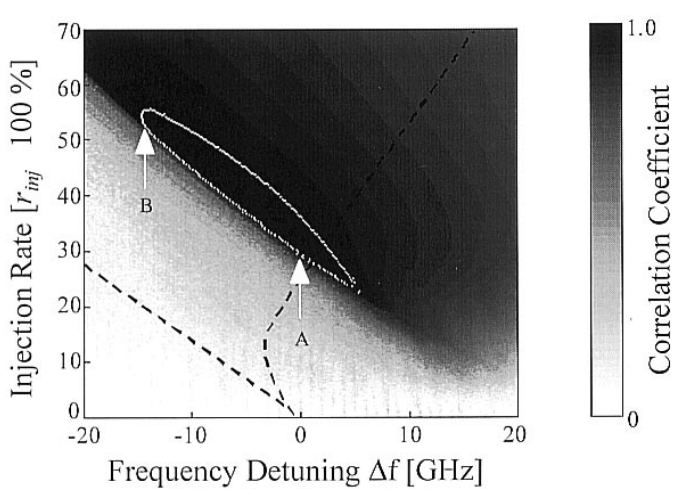

(c)

Fig. 4. Anticipating chaos synchronization region in the phase space of the frequency detuning $\Delta f$ and the optical injection rate $r_{\text {inj }}$ for strong optical feedback of $r=$ (a) 0.200 , (b) 0.242 , and (c) 0.300 . The time series corresponding to the conditions of points A and B in Fig. 4(c) are investigated and shown in Fig. 5.

feedback rate. In the previous papers, anticipating chaos synchronization has been only reported for a small injection rate at zero-frequency detuning. [12] Namely, anticipating synchronization is exactly coincident with complete chaos synchronization when the optical injection rate balances with the fraction of the optical feedback at zero-frequency detuning. However, anticipating chaos synchronization is also achieved at higher injection rate and, furthermore, it is attained even for nonzero-frequency detuning.

Anticipating or complete chaos synchronization only occurs under the condition of zero parameter-mismatch in a strict sense, namely $100 \%$ correlation. Allowing errors of the correlation coefficient, we defined anticipating chaos synchronization. Though the correlation is not perfect, the 


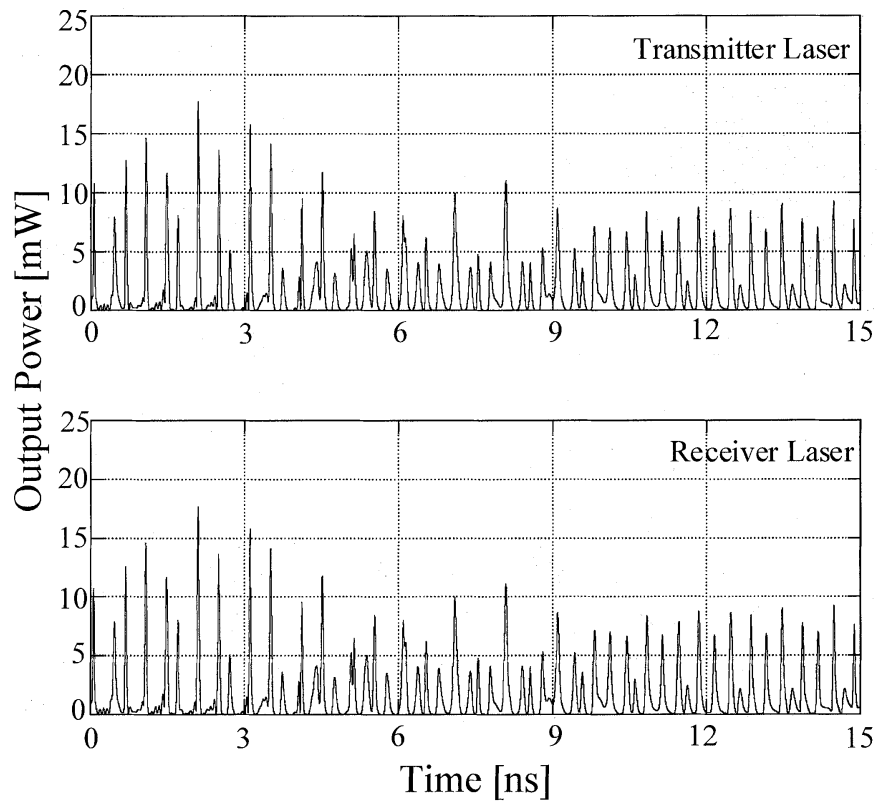

(a)

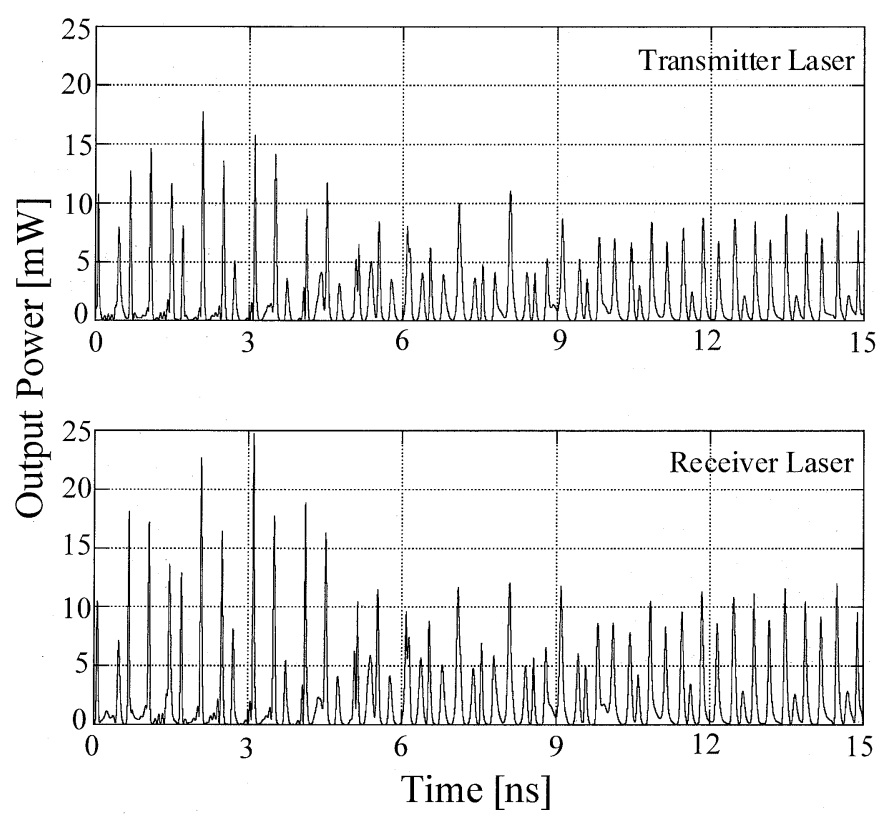

(b)

Fig. 5. (a) Time series of the transmitter and receiver lasers under the condition of point A in Fig. 4(c). (b) Time series for point B in Fig. 4(c). The time lag between the two signals is compensated.

output from the receiver laser shows an anticipating signal under parameter-mismatch conditions and anticipating chaos synchronization is approximately realized in these regions. The physical origin of synchronization for such a case is different from the complete scheme. To show the physical origin of the new type of anticipating chaos synchronization, we investigated time series and trajectories for complete and noncomplete cases of anticipating chaos synchronization. The parameter values we investigated are pointed by arrows A and B in Fig. 4(c). Fig. 5(a) shows time series of the outputs for the transmitter and receiver lasers at point $\mathrm{A}$. When the frequency detuning is zero and the optical injection rate is equal to the

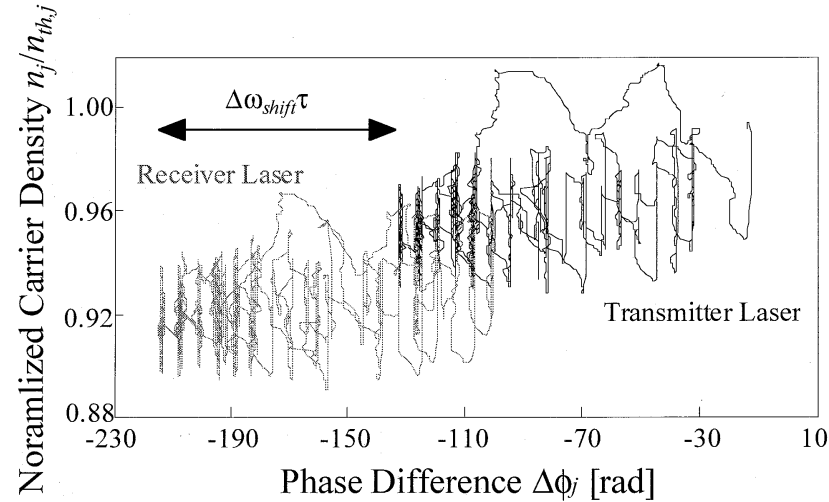

Fig. 6. Chaotic trajectories for the transmitter and receiver lasers in the phase space of the phase difference $\Delta \phi=\phi_{j}(t)-\phi_{j}\left(t-\tau_{t}\right)$ and the normalized carrier density $n_{j} / n_{\text {th }, j}$. The black line is for the transmitter laser and the gray one for the receiver laser. The conditions are the same as those for point B in Fig. 4(c). The shift of the phase difference between the trajectories of the transmitter and receiver lasers is equal to the frequency detuning.

external feedback rate, the two signals synchronize with each other under the condition of complete chaos synchronization. The two signals show almost the same oscillations not only for the waveforms but also their levels. The value of the correlation coefficient was calculated to be $C(\Delta t)=0.99$ (where $\left.\Delta t=\tau_{c}-\tau_{t}\right)$. The trajectories of chaotic itineraries of the transmitter and receiver lasers completely overlap with each other in the phase space of the phase difference $\Delta \phi=\phi_{j}(t)-\phi_{j}\left(t-\tau_{t}\right)$ and the carrier density $n_{j} / n_{\text {th }}$ (not shown here). On the other hand, in the presence of parameter mismatches, the receiver signal in Fig. 5(b) looks like quite similar to that of the transmitter but it is amplified due to a larger optical injection rate from the transmitter to the receiver though the time lag is equal to that of the anticipating scheme. The value of the correlation coefficient deteriorated a little compared with the case of the complete synchronization in Fig. 5(a), however it still maintained a high value of $C(\Delta t)=0.95$ (where $\Delta t=\tau_{c}-\tau_{t}$ ). Namely, this case of chaos synchronization is originated from an optical injection-locking phenomenon. Fig. 5(b) corresponds to the parameter conditions of point B in Fig. 4(c). The frequency detuning at point $\mathrm{B}$ was $\Delta f=-13 \mathrm{GHz}$ and the optical injection rate was $r_{\text {inj }}=0.530$.

To show explicitly the existence of amplified anticipating chaos synchronization, we plot the trajectories for the time series of Fig. 5(b) in the phase space of the phase difference $\Delta \phi=\phi_{j}(t)-\phi_{j}\left(t-\tau_{t}\right)$ and the normalized carrier density $n_{j} / n_{\mathrm{th}, j}$. Fig. 6 is the result. The trajectory of the receiver laser (gray line) is quite similar to that of the transmitter (black line), but they are separated both for the phase difference and the carrier density. The separation of the two trajectories along the phase axis corresponds to the phase difference due to the frequency detuning between the two lasers $(81.7 \mathrm{rad}$ corresponding to the frequency detuning of $-13 \mathrm{GHz}$ ). Thus, the receiver laser imitates the transmitter output as behaving as anticipating chaos synchronization on the time lag, but the synchronization is originated from optical injection-locking or amplification in laser systems. As usual, when chaos synchronization comes from optical injection-locking phenomenon, the 
receiver laser responds immediately after it receives a chaotic signal from the transmitter and the receiver signal always has a time delay with respect to the transmitter signal equal to the time of light transmission from the transmitter to the receiver, i.e., the generalized chaos synchronization scheme. Therefore, the observed results here are quite different from previous chaos synchronization as a physical origin.

\section{CONCLUSION}

Anticipating chaos synchronization in semiconductor laser systems was usually regarded as the same phenomenon as complete chaos synchronization. However, we have numerically demonstrated in the systems of semiconductor lasers with optical feedback that there were solutions for anticipating chaos synchronization with injection-locking origin when the optical feedback in the transmitter system was high enough. We have shown the evidence of anticipating chaos synchronization with optical injection-locking by showing chaotic time series and trajectories in the phase space. The region of anticipating chaos synchronization increased with the increase of the optical feedback and it lay across the stable injection-locking boundary. Namely, even when the two laser systems had parameter mismatches, anticipating chaos synchronization occurred in the nonlinear systems. Therefore, it is concluded that anticipating chaos synchronization is not a unique feature only for complete chaos synchronization in nonlinear systems. These results give rise to important information for the applications of chaos synchronization such as for chaotic secure communications.

\section{ACKNOWLEDGMENT}

The authors would like to thank Y. Takiguchi for helpful discussion.

\section{REFERENCES}

[1] J. Ohtsubo, "Chaotic dynamics in semiconductor lasers with optical feedback," in Progress in Optics, E. Wolf, Ed. Amsterdam, The Netherlands: North-Holland, 2002, vol. 44, ch. 1, pp. 1-84.

[2] - "Chaos synchronization and chaotic signal masking in semiconductor lasers with optical feedback," IEEE J. Quantum Electron., vol. 38, pp. 1141-1154, Sept. 2002.

[3] C. Masoller, "Anticipation in the synchronization of chaotic semiconductor lasers with optical feedback," Phys. Rev. Lett., vol. 86, pp. 2782-2785, 2001.

[4] Y. Liu, Y. Takiguchi, P. Davis, T. Aida, S. Saito, and J. M. Liu, "Experimental observation of complete chaos synchronization in semiconductor lasers," Appl. Phys. Lett., vol. 80, pp. 4306-4308, 2002.

[5] V. Ahlers, U. Parlitz, and W. Lauterborn, "Hyperchaotic dynamics and synchronization of external-cavity semiconductor lasers," Phys. Rev. E, vol. 58, pp. 7208-7213, 1998.

[6] A. Locquet, F. Rogister, M. Sciamanna, M. Mégret, and P. Blondel, "Two types of synchronization in unidirectionally coupled chaotic externalcavity semiconductor lasers," Phys. Rev. E, vol. 64, pp. 045 203-1-4, 2001.

[7] Y. Liu, H. F. Chen, J. M. Liu, P. Davis, and T. Aida, "Synchronization of optical-feedback-induced chaos in semiconductor lasers by optical injection," Phys. Rev. A, vol. 63, pp. 031 802(R)-1-4, 2001.

[8] I. V. Koryukin and P. Mandel, "Two regimes of synchronization in unidirectionally coupled semiconductor lasers," Phys. Rev. E, vol. 65, pp. 026201-1-5, 2002.

[9] R. Vicente, T. Pérez, and C. R. Mirasso, "Open-versus closed-loop performance of synchronized chaotic external-cavity semiconductor lasers," IEEE J. Quantum Electron., vol. 38, pp. 1197-1204, Sept. 2002.
[10] A. Locquet, C. Masoller, P. Mégret, and M. Blondel, "Comparison of two types of synchronization of external-cavity semiconductor lasers," Opt. Lett., vol. 27, pp. 31-33, 2002.

[11] A. Locquet, C. Masoller, and C. R. Mirasso, "Synchronization regimes of optical-feedback-induced chaos in unidirectionally coupled semiconductor lasers," Phys. Rev. E, vol. 65, pp. 056 205-1-12, 2002.

[12] A. Murakami and J. Ohtsubo, "Synchronization of feedback-induced chaos in semiconductor lasers by optical injection," Phys. Rev. A, vol. 65, pp. 033 826-1-7, 2002.

[13] I. Fischer, Y. Liu, and P. Davis, "Synchronization of chaotic semiconductor laser dynamics on subnanosecond time scales and its potential for chaos communication," Phys. Rev. A, vol. 62, pp. 011 801-1-4, 2000.

[14] Y. Takiguchi, H. Fujino, and J. Ohtsubo, "Experimental synchronization of chaotic oscillations in external cavity semiconductor lasers in lowfrequency fluctuation regime," Opt. Lett., vol. 24, pp. 1570-1572, 1999.

[15] H. Fujino and J. Ohtsubo, "Experimental synchronization of chaotic oscillations in external-cavity semiconductor lasers," Opt. Lett., vol. 25, pp. $625-627,2000$

[16] - "Synchronization of chaotic oscillations in mutually coupled semiconductor lasers," Opt. Rev., vol. 8, pp. 351-357, 2001.

[17] S. Sivaprakasam and K. A. Shore, "Demonstration of optical synchronization of chaotic external cavity semiconductor lasers," Opt. Lett., vol. 24, pp. 466-468, 1999

[18] M. Peil, T. Heil, I. Fischer, and W. Elsä ßer, "Synchronization of chaotic semiconductor laser systems: A vectorial coupling-dependent scenario," Phys. Rev. Lett., vol. 88, pp. 174 101-1-4, 2002.

[19] F. Rogister, A. Locquet, D. Pieroux, M. Sciamanna, O. Deparis, P. Mégret, and M. Blondel, "Secure communication scheme using chaotic laser diodes subject to incoherent optical feedback and incoherent optical injection," Opt. Lett., vol. 26, pp. 1489-1491, 2001

[20] F. Rogister, D. Pieroux, M. Sciamanna, P. Mégreta, and M. Blondel, "Anticipating synchronization of two chaotic laser diodes by incoherent optical coupling and its application to secure communications," Opt. Commun., vol. 207, pp. 295-306, 2002.

[21] S. Tang, H. F. Chen, and J. M. Liu, "Stable route-tracking synchronization between two chaotically pulsing semiconductor lasers," Opt. Lett., vol. 26, pp. 1489-1491, 2001.

[22] S. Tang and J. M. Liu, "Chaos synchronization in semiconductor lasers with optoelectronic feedback," IEEE J. Quantum Electron., vol. 39, pp. 708-715, June 2003.

[23] H. U. Voss, "Anticipating chaotic synchronization," Phys. Rev. E, vol. 61 , pp. 5115-5119, 2000.

Kenji Kusumoto was born in Nagasaki, Japan, in 1977. He received the B.S. degree in electronics from University of Industrial Technology, Kanagawa, Japan, in 2001 and the M.S. degree in systems engineering from Shizuoka University, Hamamatsu, Japan, in 2003.

$\mathrm{He}$ is now working for Yokogawa Electric Company, Tokyo. His research interests are synchronization of optical feedback-induced chaos in semiconductor lasers.

Mr. Kusumoto is a Member of the Japanese Society of Applied Physics.

Junji Ohtsubo (M'96) received the B.S. degree in electronics from the Kyushu Institute of Technology, Kyushu, Japan, in 1973 and the M.S. and Ph.D. degrees in electronics from Hokkaido University, Hokkaido, Japan, in 1975 and 1978, respectively.

In 1978, he joined the Mechanical Engineering Laboratory, MITI, Japan. During 1981-1982, he was a Research Associate at the Institute of Optics, University of Rochester, Rochester, NY. He joined Shizuoka University, Hamamatsu, Japan, as an Associate Professor in 1985 and is presently a Professor. His current research interests are nonlinear dynamics in optics, optical information processing and computing, optical security systems, statistical optics, speckle, and optical metrology.

Prof. Ohtsubo is a Fellow of the Optical Society of America and a Member of SPIE, the American Physical Society, the Japanese Society of Applied Physics, the Optical Society of Japan, and the Laser Society of Japan. 